\title{
La formación en competencias como estrategia para mejorar la dirección pública
}

\author{
Ana M. Castaño y Antonio L. García-Izquierdo \\ Universidad de Oviedo, (España)
}

\begin{abstract}
Ana María Castaño ha participado en el proyecto Evaluación y Selección de Personal Directivo Público (Proyecto ESPUMA: https://espuma.uniovi.es/) en la Universidad de Oviedo. Actualmente, trabaja como investigadora en la Universidad de Oviedo y su línea de investigación se centra en liderazgo público, selección y evaluación, justicia organizacional e igualdad de oportunidades en el empleo entre mujeres y hombres.
\end{abstract}

ORCID: https://orcid.org/0000-0003-1659-7625 castanoperez.ana@gmail.com

Antonio León García-Izquierdo es profesor titular y director de la Cátedra Asturias Prevención de la Universidad de Oviedo. Su línea de investigación se centra en la selección y evaluación, la igualdad de oportunidades en el empleo entre mujeres y hombres y los riesgos psicosociales en el trabajo. Es el investigador principal del proyecto Evaluación y Selección de Personal Directivo Público (Proyecto ESPUMA: https://espuma.uniovi.es/).

ORCID: https://orcid.org/0000-0002-2515-3439 angarcia@uniovi.es

\section{RESUMEN}

La reciente crisis económica ha acrecentado la preocupación por un servicio público eficiente, haciendo necesario mejorar las prácticas de gestión para afrontar los desafíos organizacionales emergentes como la diversidad, la ética y la digitalización, entre otros. Para ello, un elemento crucial es la formación para el desarrollo de competencias directivas que mediante la modalidad blended learning conforma una estrategia para mejorar la dirección pública. Esta modalidad permite integrar formación presencial y a distancia mediante las plataformas MOOC reduciendo costes y flexibilizando tiempos.

El objetivo de este trabajo es elaborar una propuesta fundamentada desde el enfoque de aprender haciendo para desarrollar programas formativos para el personal directivo. Primero, hemos analizado las limitaciones de los procesos de recursos humanos, proponiendo los modelos de competencias como un marco adecuado para este fin. Posteriormente, tras la detección de necesidades formativas hemos propuesto un programa que incluye estrategias formativas y evaluativas para las denominadas competencias duras y blandas. Finalmente, hemos discutido las implicaciones del programa formativo en el contexto actual de los constantes cambios tecnológicos y sociales.

Palabras Clave: competencias, personal directivo público, formación, justicia organizacional, blended learning.

\section{Introducción}

La reciente crisis económica ha puesto en evidencia el deterioro de la Administración Pública (AP) española, de modo que podría resumirse, siguiendo a \iménez (2018), en: (i) un personal envejecido con dificultades para abordar una transformación digital, (ii) un nivel tecnológico inferior al deseado en la era de la información y las comunicaciones, y (iii) una anomia de valores con un número creciente de casos de corrupción, lo que ha incrementado la preocupación por la prestación eficiente de los servicios públicos. Por lo tanto, la AP necesita mejorar y modernizar sus prácticas de gestión (e.g., Instituto Nacional de Administración Pública - INAP, 2014) para afrontar los desafíos organizacionales emergentes, como la diversidad, la ética, y la digitalización, entre otros. Para lograr este propósito, dada la relevante posición del personal directivo debido a la calidad de sus decisiones, un elemento crucial es el desarrollo de sus competencias (e.g., Berman y West, 2003), y una manera eficaz de lograrlo es mediante la formación. Una modalidad de aprendizaje útil para la formación del personal directivo es el blended learning. Esta modalidad permite combinar la formación y el coaching presencial y a distancia mediante los cursos masivos abiertos en línea (Massive Open Online Courses - MOOC) que facilita el desarrollo de competencias a la vez que reduce costes, flexibiliza tiempos y acerca el uso de las tecnologías de la información y las comunicaciones al personal directivo (e.g., Holland y Piper, 2016; Singer y Stoicescu, 2011; Singh, 2003). Tomando en consideración lo anterior, el objetivo de este trabajo es elaborar una propuesta fundamentada en el enfoque de aprender haciendo (Dewey, 1926; Schank, 2002) para desarrollar programas de formación del personal directivo en la AP.

Teniendo en cuenta este objetivo, primero, se ha analizado las limitaciones de los procesos de recursos humanos y se ha propuesto los modelos de competencias como un marco adecuado 
para mejorar la dirección pública. Posteriormente, se han detectado las necesidades de formación actuales y futuras y se ha propuesto un programa de formación en competencias. Finalmente, se han discutido sus implicaciones dentro del contexto actual de continuos cambios tecnológicos y sociales.

\section{Los desafíos para la gestión de los recursos humanos en la AP española}

Se considera personal directivo público a quienes realizan funciones directivas, según el artículo 13 del Real Decreto Legislativo 5/2015, de 30 de octubre, por el que se aprueba el texto refundido de la Ley del Estatuto Básico del Empleado Público (EBEP). Se refiere tanto a posiciones que desempeñan funciones de dirección y tienen personal a su cargo, como a personal directivo de alto rango nombrado mediante criterios de confianza personal y/o política. Estos diferentes tipos de personal directivo acceden por procesos diferentes. Por un lado, los directivos que acceden como funcionarios de carrera deben superar oposiciones mediante, principalmente, pruebas de conocimiento o una combinación de estas con las evaluaciones de méritos. Posteriormente, los procesos de promoción son muy similares, no obstante, son necesarios dos años de experiencia en el cargo para poder optar a dicha promoción. En cuanto a las limitaciones de estos procesos, en el caso de las pruebas de conocimiento hay una falta de conexión percibida entre los contenidos y las tareas (Rodríguez, 2013), y la evidencia empírica sobre su adecuación con el criterio de igualdad es escasa (Salgado y Moscoso, 2008). Respecto a las evaluaciones de méritos, Fondevila (2012) argumenta que podrían violar los principios de igualdad, mérito, capacidad y equidad porque: (i) los políticos podrían favorecer a sus candidatos preferidos al exigir méritos específicos (Ruano, Crespo, y Polo, 2014), (ii) discriminan indirectamente a las mujeres (Alonso, Táuriz, y Choragwicka, 2009), y de forma específica, (iii) las preguntas invasivas podrían conducir a la discriminación por género (e.g., García-Izquierdo, Ramos-Villagrasa, y Castaño, 2015) ya que pueden ser vistas como activadores de los estereotipos (Castaño, Fontanil, y García-Izquierdo, 2019).

Por otro lado, algunos altos cargos pueden ser seleccionados a través de la libre designación, ya que, por ejemplo, en muchas comunidades autónomas todavía no se ha desarrollado detalladamente la regulación sobre la selección de su personal directivo (e.g., Areses et al., 2017). Aquí, surge otra limitación respecto a la prioridad otorgada a la lealtad política sobre las competencias (e.g., Bach y Veit, 2017). Como señala ఏiménez (2009), si el único criterio de selección es la confianza política, esta discreción en la designación puede implicar una violación de los principios de igualdad, equidad, mérito y capacidad. Además, la posible falta de profesionalidad por no considerar las competencias directivas para su selección podría afectar la eficacia de la gestión y de las decisiones por parte de la dirección (e.g., Sánchez, 2018).

Como resultado de estas limitaciones, se podría percibir a la $\mathrm{AP}$ como una organización que presenta muchas dificultades para afrontar los desafíos organizacionales actuales anteriormente expuestos. Por lo tanto, la AP española debe promover su modernización aplicando, entre otras, las líneas del Gobierno Abierto, la transparencia y la igualdad en el empleo como una oportunidad para recuperar la confianza de los ciudadanos.

\section{Gobierno Abierto e igualdad}

El Gobierno Abierto consiste en la aplicación de los principios de rendición de cuentas y la promoción de la transparencia, la participación, y la colaboración entre la ciudadanía y la AP de manera justa (García, 2014). Para lograr un Gobierno Abierto, son necesarios varios y diversos cambios en distintos aspectos, como los que se citan a continuación: (i) los aspectos culturales, para comprender que el objetivo principal de la AP es servir a los ciudadanos; (ii) la propia organización, que está diseñada bajo sistemas jerárquicos y no siempre relacionados con la eficiencia; y (iii) las relaciones entre los ciudadanos y la AP para conseguir una comunicación más participativa y un sistema más abierto y transparente, que facilite el manejo de información, lo que requiere el desarrollo y uso de tecnologías para comunicar esa información a la ciudadanía.

Por otro lado, cuando los trabajadores perciben a una organización como socialmente responsable, estos responden con un mejor desempeño, compromiso, atracción organizacional y actitudes positivas hacia el trabajo (e.g., Rupp, Wright, Aryee, y Luo, 2015). En esta línea, el desarrollo de la Ley Orgánica 3/2007 de 22 de marzo para la igualdad efectiva entre mujeres y hombres, $y$ del EBEP es hacer efectivo el derecho a la igualdad de trato y de oportunidades mediante la eliminación de la discriminación hacia la mujer en las organizaciones. En este mismo sentido, tanto la política de Gobierno Abierto como la de igualdad de oportunidades están estrechamente relacionadas con evitar la corrupción y promover la ética. Esto se debe a que la corrupción no solo ocurre cuando quien ocupa un cargo público lo utiliza para obtener un beneficio personal, sino también cuando hay una mala gestión de los recursos públicos prolongada en el tiempo, incluso aunque no se busque el propio beneficio_(Jareño, 2011). A este respecto, Naessens (2010) señala que los empleados públicos que reciben formación adecuada, son más firmes para hacer frente a la corrupción.

Ante estos desafíos, los enfoques tradicionales de recursos humanos ya no son suficientes por lo que se precisan enfoques centrados en competencias orientadas hacia un contexto más volátil y cambiante (e.g., Martínez-González, Selva, y Crespo, 2019). En estos nuevos enfoques, destaca el liderazgo transformacional (Bass, 1985), según el cual el personal directivo debe crear un cambio hacia el desarrollo personal de los empleados dentro de la organización; y el liderazgo ético, que alienta a los empleados a adoptar un repertorio de comportamientos que evite la corrupción (e.g., Stouten, van Dijke, y De Cremer, 2012). Específicamente, siguiendo a Ramió y Salvador (2018), si la AP pretende ser eficiente y efectiva en la prestación de servicios debe contar con profesionales competentes con vocación de servicio. En consecuencia, es necesario identificar y detectar nuevos perfiles de competencias y su evaluación (e.g. Jiménez, 2018) mediante modelos de selección y promoción rigurosos que utilicen pruebas fiables y válidas que puedan garantizar evaluaciones eficaces y justas en los procesos de selección y de promoción de acuerdo con los principios de mérito, capacidad, publicidad e igualdad (e.g., García-Izquierdo y García-Izquierdo, 2007; García-Izquierdo, Moscoso, y Ramos-Villagrasa, 2012; Rodríguez y López-Basterra, 2018) por lo que un modelo de recursos humanos basado en competencias facilitaría la modernización de las instituciones públicas, al tiempo que ofrecería un marco adecuado para un desempeño exitoso y ecuánime.

\section{Los modelos de competencias}

Una amplia variedad de definiciones para el término competencia ha surgido desde que $\mathrm{McClelland}$ (1973) destacara que se refiere a una diferencia crítica en los comportamientos de las personas que tienen un desempeño exitoso. Ahondando en ello, son características medibles del repertorio conductual que 
contribuyen de manera diferencial al desempeño laboral (e.g.. Bartram, 2005; Salgado y Cabal, 2011). De acuerdo con Armstrong (2006), un modelo basado en competencias es útil para mejorar los procesos de reclutamiento, selección, y la gestión del desempeño. Teniendo en cuenta su utilidad, no es sorprendente que se haya desarrollado un gran número de modelos de competencias tanto en organizaciones públicas como privadas (e.g., Ennis, 2008; United Kingdom Civil Service - marco del Servicio Civil del Reino Unido, 2015). Esto se debe también, entre otras razones, a que los modelos de competencias proporcionan un lenguaje común para las organizaciones (Briscoe y Hall, 1999). Asimismo, pueden resultar apropiados para: (i) mejorar la justicia organizacional (e.g., Cropanzano, Bowen, y Gilliland, 2007); (ii) lograr validez aparente en los procesos de evaluación (e.g., Truxillo y Bauer, 2011); y (iii) alcanzar la diversidad (e.g., Castaño et al., 2019) en los procesos de recursos humanos. Además, estos modelos permiten identificar y detectar las competencias requeridas por el personal directivo para orientar su formación (e.g., De Waal, 2010) cuando se accede al cargo desde posiciones de menor rango y responsabilidad, donde las competencias pueden ser distintas (e.g., Park y Faerman, 2018). Este aspecto es especialmente relevante en el caso de la AP es-

Tabla 1

Etapas para el desarrollo de un programa de formación en competencias directivas pañola, ya que de acuerdo con los artículos 14 y 69 del EBEP, la formación es un derecho de los empleados públicos y una herramienta para contribuir a proporcionar servicios de manera efectiva, por lo que, a continuación, se presentará la propuesta de un programa de formación en competencias para el personal directivo público.

\section{Desarrollo de un programa de formación para el personal di- rectivo público basado en competencias}

El desarrollo de un programa formativo es un proceso sistemático (Berrocal y Pereda, 1999). En el caso del personal directivo, en primer lugar, se debe identificar y detectar las necesidades formativas en términos de competencias; en segundo lugar, los programas de formación deben diseñarse y llevarse a cabo de acuerdo con las necesidades de formación, estableciendo para ello las modalidades y metodologías de aprendizaje que mejor se adecuen al personal directivo y su contexto organizacional; $y$, en tercer lugar, se debe evaluar el programa. La Tabla 1 proporciona un resumen de estas etapas, que se detallarán en las secciones siguientes.
Etapa del programa
Descripción de las etapas

Pasos para la identificación y detección de necesidades formativas

- Elaboración de un modelo preliminar de competencias.

- Sesiones de grupos focales y paneles Delphi con expertos para refinar el modelo.

Identificación y detección de - Análisis y validez del modelo respecto al desempeño laboral.

las necesidades formativas

Resultados: un modelo de ocho competencias

- Competencias duras: planificación, liderazgo, orientación a la innovación, y orientación al servicio público.

- Competencias blandas: reconocimiento y regulación de emociones, compromiso con la AP, comunicación y ética.

Modalidades y metodologías

- Evaluación/certificación inicial mediante assessment centers.

- Formación en competencias mediante blended-learning utilizando MOOC desde metodologías activas:

- Formación teórica: aulas virtuales.

- Formación a distancia: simulaciones y resolución de problemas; proporcionando un canal para contactar con

Diseño y ejecución mentores y otros participantes (e.g., reuniones en línea, mensajes, foros).

- Formación presencial: talleres prácticos y asesoramiento por parte de directivos públicos con una amplia experiencia profesional.

Otros aspectos que deben considerarse

- Formación adaptada a la discapacidad visual y/o auditiva.

- Cumplimiento de la legislación en materia de protección de datos y prevención de riesgos laborales.

\section{¿Qué información se debe recopilar y cuándo?}

- Reacciones, aprendizaje, comportamiento y resultados: inmediatamente después de la formación y pasado un tiempo para analizar la estabilidad de los cambios.

Evaluación - Actualización de las necesidades formativas y nivel de competencias: periódicamente después de la formación.

$$
\text { ¿Cómo obtener la información para la evaluación? }
$$

- Assessment centers, para los niveles de competencia, el aprendizaje y el comportamiento.

- Cuestionarios de satisfacción, para las reacciones de los participantes.

- Evaluaciones de desempeño 360ㅜㅡ, número de quejas, satisfacción de subordinados, para los resultados.

\section{La identificación y detección de las necesidades formativas}

El primer paso para desarrollar un programa de formación es identificar y detectar las necesidades formativas para posteriormente establecer las metodologías y modalidades de apren- dizaje más eficientes (e.g., Sladogna, 2003). A este respecto, para la identificación y detección de las competencias que el personal directivo necesita desarrollar, proponemos seguir el enfoque híbrido de Briscoe y Hall (1999), que permite tener en cuenta los requisitos actuales y futuros de la organización combinando in- 
vestigación cualitativa y cuantitativa. En la Tabla 2 se recogen algunos ejemplos de los pasos específicos para desarrollar un modelo de competencias directivas que se enmarcan en este enfoque híbrido. Estos ejemplos tienen en común la participación de expertos (i.e., expertos en recursos humanos y empleados con un profundo conocimiento sobre el puesto de trabajo y la organización), la revisión de otros modelos de competencias y la validación del modelo en términos de desempeño laboral.

Tabla 2

Resumen de los principales pasos para desarrollar un modelo de competencias directivas

Draganidis y Mentzas (2006)

(i) Identificación y contacto con el grupo de expertos.

(ii) Identificación de la muestra y las medidas de desempeño para la validación.

(iii) Desarrollo de un listado inicial competencias mediante la revisión de modelos existentes.

(iv) Definición de competencias e indicadores de comportamiento mediante la realización de entrevistas, grupos focales y encuestas; y (v) desarrollo de un modelo de competencia inicial a través del análisis cuantitativo y de contenido del paso anterior.

(vi) Validación cruzada mediante grupos focales, entrevistas y/o encuestas; y (vii) perfeccionamiento del modelo, a través del análisis cuantitativo y de contenido de esta validación.

(viii) Validación del modelo, convirtiendo las competencias en un cuestionario.

(ix) Finalización del modelo, eliminando cualquier competencia que no correlacione con el desempeño.

Para ilustrar esta fase de identificación y detección, a continuación se resume cómo se ha desarrollado un modelo de competencias directivas siguiendo el enfoque híbrido anteriormente citado dentro del marco de investigación del proyecto Evaluación y Selección de Personal Directivo Público (ESPUMA ${ }^{1}$ ). En primer lugar, se realizó un análisis de contenido de la literatura sobre competencias directivas públicas que permitió obtener un modelo de competencias preliminar. En segundo lugar, se llevaron a cabo varias sesiones de grupos focales con expertos, y la cumplimentación de un cuestionario para refinar el modelo preliminar. En tercer lugar, el modelo se perfeccionó en un cuestionario definiendo las competencias en términos de descriptores comportamentales. En cuarto lugar, el cuestionario se refinó llevando a cabo un panel Delphi con expertos donde se pidió a los participantes que: (i) priorizaran las competencias en función de su relación con el desempeño directivo público razonando sus elecciones; (ii) indicaran su grado de acuerdo con la lista de competencias; (iii) indicaran su acuerdo con la nueva lista; y (iv) proporcionaran sugerencias finales. $Y$, en quinto lugar, se analizaron las propiedades psicométricas del cuestionario de competencias y se probó su validez en relación con el desempeño laboral con una amplia muestra de personal directivo público. Como resultado, se desarrolló un modelo de ocho competencias directivas que incluye: competencias duras como la planificación, el liderazgo, la orientación a la innovación y la orientación al servicio público, y competencias blandas como el reconocimiento y la regulación de las emociones, el compromiso con la $\mathrm{AP}$, la comunicación, y la ética.

Una vez identificadas y detectadas las competencias que debe desarrollar el personal directivo, la siguiente etapa es el diseño y propuesta del programa formativo para su desarrollo.
Czabanowska et al. (2013) y Tett, Guterman, Bleier, y Murphy (2000)

(i) Revisión de la literatura y/o modelos existentes para obtener un modelo preliminar de competencias.

(ii) Mejora del modelo preliminar mediante su evaluación por parte de expertos.

(iii) Validación del modelo mediante envío de cuestionarios y/o realización de paneles Delphi.

Los programas de formación del personal directivo público: diseño y ejecución de una propuesta

Varios son los programas de formación para personal directivo público que se han desarrollado en España. El INAP tiene un programa específico para directivos públicos con experiencia y para quienes están en posiciones pre-directivas. También las escuelas de negocios (e.g., IESE, ESADE), la Federación Española de Municipios y Provincias y las escuelas de AP de las comunidades autónomas ofrecen programas de formación para directivos públicos, además de las numerosas universidades españolas que ofrecen programas de formación para la dirección pública. Pero, a pesar de las ventajas de estos programas formativos, desafortunadamente también poseen algunas limitaciones. Una primera limitación se refiere al carácter voluntario de estos programas. Una segunda limitación se refiere a la pérdida de saber hacer del personal directivo con experiencia debido a la falta de políticas de gestión del conocimiento. Una tercera limitación se refiere a la falta de una evaluación de competencias previa al inicio de la formación para elaborar un programa a medida. Por ejemplo, mediante la certificación de competencias adquiridas durante el ejercicio de cargos directivos.

Más allá de estas limitaciones, se propone a continuación un programa de formación para el desarrollo de competencias directivas. Este programa está basado en el modelo del proceso de enseñanza-aprendizaje sugerido por de Miguel (2006), donde el centro de atención para la planificación de las modalidades, métodos y evaluación del programa lo ocupan las competencias que se deben desarrollar.

En primer lugar, el programa de formación propuesto incluye una etapa inicial para la evaluación/certificación de las competencias a través de evaluaciones del comportamiento,

Para más información: https://espuma.uniovi.es/ 
como por ejemplo, los assessment centers. Esta evaluación/certificación incluye, entre otras, pruebas de juicio situacional y muestras de trabajo, en las que se reconstruyen los entornos de trabajo para evaluar los comportamientos (e.g., Weekley, Hawkes, Guenole, y Ployhart, 2015) y que son especialmente adecuadas dada su validez aparente y de criterio (e.g., Anderson y Witvliet, 2008; Christian, Edwards, y Bradley, 2010). Llevar a cabo una evaluación/certificación inicial de las competencias mejora la idoneidad del programa, ya que se centra específicamente en: (i) las competencias que requieren de formación, (ii) las necesidades específicas de cada nivel directivo, y (iii) las necesidades concretas según el nivel de AP (i.e., AP Local, Autónoma y Estatal).

Tras esta evaluación/certificación inicial, la propuesta incluye un programa de formación que parte de la modalidad de blended-learning, donde se combina la modalidad presencial con el trabajo autónomo y la formación a distancia. Esta modalidad ha demostrado su efectividad en cuanto al desempeño y la satisfacción de los participantes (e.g., Ho, Lu, y Thurmaier, 2006; Umek, Aristovnik, Tomaževič, y Keržič, 2015), así como su idoneidad para la docencia de competencias (Singer y Stoicescu, 2011). Además, el blended-learning contribuye a la apertura al e-learning, escasamente introducido en la AP (Stoffregen, Pawlowski, y Pirkkalainen, 2015), al aprovechar tanto el aprendizaje presencial como las plataformas MOOC. La flexibilidad de combinar estas dos modalidades de aprendizaje también permite la participación de aquellos que no pueden asistir a la formación en un momento específico, así como el aprendizaje desde el enfoque de la formación en el puesto, cercano al enfoque de aprender haciendo. En cuanto a las estrategias metodológicas para la formación (e.g., de Miguel, 2006), el programa propuesto sigue un diseño modular que facilita la ejecución solamente de aquellos módulos necesarios en función de los resultados de la evaluación/certificación inicial de las competencias. No obstante, esta propuesta se complementa con otros métodos especialmente adecuados para el desarrollo de competencias donde encajan tanto el método expositivo, como otras metodologías activas como son el estudio de casos, las tutorías, las simulaciones, la resolución de problemas y los serious games. Estas metodologías, que van en línea con la tradición de aprender haciendo, permiten formar para enfrentar nuevas situaciones con éxito (e.g., Stene, 1970) haciendo uso de las nuevas tecnologías para la formación en competencias de una manera atractiva (e.g., Korteling, Helsdingen, y Theunissen, 2013).

Por último, pero no menos importante, la propuesta formativa tiene en consideración los siguientes aspectos: (i) los materiales y plataformas MOOC se adaptan asegurando el principio de igualdad también en términos de accesibilidad visual y/o auditiva; (ii) además, el programa cumple con la legislación sobre protección de datos, especialmente al diseñar las plataformas MOOC y al realizar las evaluaciones; finalmente, (iii) y estrechamente relacionado con esto último, las instalaciones para la formación, así como el equipo para la formación a distancia garantizan el cumplimiento de la legislación en materia de prevención de riesgos laborales.

Teniendo en cuenta lo anterior, y siguiendo con el ejemplo que ha ilustrado la sección de identificación y detección de las necesidades formativas, el programa de formación propuesto para el modelo de competencias identificado incluye un módulo de blended-learning para cada competencia, con una duración que variará en función de la evaluación/certificación inicial de competencias y el nivel de desarrollo de competencias deseado. El principio rector de cada módulo es formar a los participantes para que se conviertan en personal directivo que, siguiendo las definiciones establecidas para cada competencia: (i) liderazgo: guíe, influya y desarrolle personas, gestione equipos y colabore y coopere para lograr objetivos comunes en línea con el enfoque del liderazgo transformacional; (ii) ética: se comporte de acuerdo con los principios del servicio público que garantizan los derechos de las personas; (iii) reconocimiento y regulación de las emociones: muestre la autorregulación emocional y la capacidad de detectar sus propias emociones y las de otros y sea capaz de tenerlas en cuenta; (iv) compromiso con la AP: comprende la estructura, objetivos, funcionamiento y política de la organización, actuando con lealtad; (v) comunicación: proporcione y obtenga información de manera clara, concisa, oportuna y segura; (vi) planificación: establezca y administre tiempos realistas y recursos para desarrollar proyectos, lograr objetivos y administrar una agenda; (vii) orientación al servicio público: ayude y sirva a los ciudadanos al detectar y satisfacer las necesidades de la ciudadanía; y (viii) orientación a la innovación: desarrolle y proponga acciones, soluciones y nuevas formas de organización y mejoras. Partiendo de estas premisas, el programa formativo comienza con una breve formación teórica sobre los comportamientos relacionados con las competencias a través de las plataformas MOOC. Para este propósito, la grabación de eventos en vivo y las aulas virtuales son de utilidad. Posteriormente, las metodologías activas de aprendizaje desde el enfoque del aprender haciendo, como las simulaciones y la resolución de problemas, se utilizan para la formación práctica a distancia. Todo esto siempre facilitando un canal para contactar a los formadores en caso de dudas mediante reuniones en línea, mensajes y foros de discusión con otros participantes del programa. En cuanto a las simulaciones, se usan pruebas de juicio situacional y muestras de trabajo, que se complementan con el uso de los serious games y las actividades de resolución de problemas. Finalmente, la formación a distancia se combina con formación presencial a través de talleres prácticos donde los participantes pueden reunirse para compartir y debatir sobre experiencias de trabajo; y con el el coaching por parte del personal directivo público con amplia experiencia para una adecuada gestión del conocimiento.

\section{La evaluación del programa de formación}

Por último, tras la ejecución del programa de formación, la siguiente etapa es su evaluación, para lo cual se propone tener en cuenta qué información debe recopilarse, cuándo y cómo recopilar dicha información.

En relación con el qué y el cuándo, por un lado, siguiendo los niveles del New World Kirkpatrick Model (Kirkpatrick y Kirkpatrick, 2016) se propone la evaluación de los siguientes aspectos: (i) las reacciones, esto es, el grado en que los participantes en el programa de formación son favorables al programa y lo encuentran atractivo y relevante; (ii) el aprendizaje, es decir, el grado en que se adquiere el conocimiento, las habilidades, la actitud, la confianza y el compromiso previstos; (iii) el comportamiento, referido al grado en que se aplica en el trabajo lo aprendido en el programa de formación y (iv) los resultados, es decir, el grado en que se producen resultados específicos esperados como consecuencia de la formación. Para obtener la información sobre estos cuatro aspectos, lo más adecuado es hacer una evaluación después de la ejecución del programa de formación y repetirla pasado un tiempo para analizar la estabilidad de los cambios obtenidos con el programa. Además, sería recomendable evaluar el aprendizaje, el comportamiento y los resultados también antes de la ejecución del programa con la finalidad de valorar en qué medida la formación ha sido efectiva. 
Por otro lado, se debe evaluar la idoneidad del programa periódicamente para valorar la posibilidad de modificar su modalidad, metodologías de aprendizaje y las competencias a desarrollar dado el contexto actual de continuos cambios. Asimismo, no basta con que el personal directivo participe en el programa formativo una vez a lo largo de su carrera profesional, sino que se propone la realización de evaluaciones periódicas de su nivel de competencias para mantener actualizada la formación garantizando su desarrollo profesional a lo largo del tiempo. En definitiva, la evaluación del programa formativo ha de resultar útil no solo para valorar su eficacia, sino también para guiar futuros programas de formación mejorando la disposición a participar del personal directivo (e.g., Dysvik y Kuvaas, 2008) al mismo tiempo que se garantiza la corrección de problemas y la anticipación a las nuevas necesidades formativas.

Respecto a cómo obtener la información mencionada anteriormente, los assessment centers, las pruebas de juicio situacional y las muestras de trabajo son apropiadas para la evaluación del aprendizaje y de los comportamientos. También, los cuestionarios de satisfacción relacionados con el contenido de la formación, su duración y las modalidades y metodologías de aprendizaje utilizadas son adecuados para la evaluación de las reacciones hacia el programa formativo. Además, las evaluaciones de desempeño $360^{\circ}$, la satisfacción de los subordinados con el personal directivo, y/o el análisis del número de quejas por parte de los ciudadanos respecto a la prestación de servicios públicos, entre otros, son útiles para evaluar los resultados.

Siguiendo con el ejemplo de la propuesta de programa formativo para las competencias de la sección anterior, su evaluación comprende dos fases. Una fase de evaluación de las reacciones, del aprendizaje, del comportamiento y de los resultados, y una fase de revisión periódica del programa formativo y de los niveles de competencias del personal directivo. Para la primera fase, se hace uso de assessment centers, pruebas de juicio situacional y muestras de trabajo específicamente diseñados para la evaluación de las competencias. Esta evaluación se realiza antes y después de la ejecución del programa formativo y se repite pasado un tiempo para su comparación. Del mismo modo, se realizan evaluaciones de desempeño $360^{\circ}$, así como el análisis de la satisfacción de los subordinados con el personal directivo, y del número de quejas por parte de los ciudadanos respecto a la prestación de servicios públicos antes de la ejecución del programa y un tiempo después de su ejecución. Finalmente, inmediatamente después de la ejecución del programa, se aplican cuestionarios de satisfacción con la formación. La segunda fase incluye una revisión periódica de las modalidades y metodologías utilizadas. Para ello, se revisa el programa de formación en línea con las nuevas metodologías y modalidades de aprendizaje existentes, los desarrollos tecnológicos y las nuevas necesidades formativas en términos de competencias directivas. Esta revisión se complementa con una evaluación también periódica de los niveles de competencias del personal directivo que ya ha recibido la formación para la planificación de formaciones de refuerzo y actualización de las competencias.

\section{Discusión y conclusiones}

En este trabajo se ha propuesto un programa formativo para el personal directivo basado en las competencias requeridas para una gestión eficaz frente a los desafíos organizacionales de la $\mathrm{AP}$, y que por lo tanto puede contribuir a su modernización. Así pues, las principales características del programa de formación aquí propuesto se discutirán a continuación.

En primer lugar, es importante destacar la relevancia de la etapa de identificación y detección de las necesidades formativas y su actualización a lo largo del tiempo, teniendo en cuenta no solo el momento actual, sino también los cambios tecnológicos y sociales futuros para definir los contenidos del programa de formación en términos de competencias directivas (i.e., ética, liderazgo, orientación a la innovación).

En segundo lugar, la evaluación/certificación inicial de las competencias de los participantes en el programa formativo reduce los tiempos y los costes, ajustando el programa a la medida de las necesidades específicas.

En tercer lugar, y en línea con lo anterior, dadas las características particulares del personal directivo público, el blended learning y las metodologías de formación activas permiten que el programa se adapte a sus necesidades para la eficacia de la formación en el puesto de trabajo. Además, el uso de los MOOC les permite acercarse a las nuevas tecnologías, lo que los acerca a su vez a los principios del Gobierno Abierto. También, la flexibilidad del blended learning permite a las mujeres acceder a la formación teniendo en cuenta los períodos de maternidad y las responsabilidades familiares (e.g., Castaño et al., 2019). Así pues, el modelo de formación en competencias facilitaría a mujeres y hombres las mismas oportunidades para el acceso a los puestos directivos contribuyendo al cumplimiento de los principios de igualdad y justicia.

En conclusión, la implementación del programa de formación aquí propuesto basado en el blended learning puede resultar en la modernización de la AP (e.g., Moskal, Dziuban, y Hartman, 2013) mediante la flexibilidad para adaptarse a los cambios organizativos y sociales, el ahorro de tiempos y costes por su adaptación a medida, la aplicación de las nuevas tecnologías en los modelos de enseñanza-aprendizaje, y el uso de metodologías activas para la formación en el puesto de trabajo de las competencias requeridas.

Financiación: Los autores informan haber recibido el siguiente apoyo financiero para la investigación, autoría y publicación de este artículo: Ministerio de Economía y Competitividad y Fondos Sociales Europeos (referencia del proyecto PSI-201344854R), Cátedra Asturias Prevención (CATI-04-2018), y Consejería de Economía y Empleo del Gobierno del Principado de Asturias y Fondos Europeos de Desarrollo Regional (referencia FC-GRUPIN-IDI/2018/000132). 


\title{
Competency vocational training as a strategy for improving public management
}

\author{
Ana M. Castaño $\left({ }^{*}\right)$ y Antonio García-Izquierdo $\left({ }^{*}\right)$ \\ ${ }^{*}$ ) University of Oviedo, Oviedo (Spain)
}

\begin{abstract}
Ana María Castaño has participated in the Project Evaluation and Selection of Public Managers (ESPUMA project: https:/espuma.uniovi.es/) at the University of Oviedo. Currently, she works as a researcher at the University of Oviedo and her line of research focuses on public leadership, selection and evaluation, organizational justice and equal opportunities in employment between women and men.
\end{abstract}

ORCID: https://orcid.org/0000-0003-1659-7625 castanoperez.ana@gmail.com

\begin{abstract}
Antonio León García-Izquierdo is a full professor and director of the Asturias Prevention Chair of the University of Oviedo. His line of research focuses on selection and evaluation, equal employment opportunities between women and men and psychosocial risks at work. He is the principal investigator of the Evaluation and Selection of Public Managers project (ESPUMA project: https://espuma.uniovi.es/).
\end{abstract}

ORCID: https://orcid.org/0000-0002-2515-3439 angarcia@uniovi.es

\begin{abstract}
In the wake of the recent economic crisis, the concern for the efficient provision of public services has been increased making necessary to improve management practices in order to face emerging organizational challenges for diversity, ethics, and digitalization, among others. For this purpose, a crucial element is the training for the development of managerial competencies which, through the blended learning modality, shapes a strategy to improve public management. This modality allows to combine face-to-face and distance training using MOOC platforms reducing costs and making schedules more flexible.

The objective of this work is to elaborate a proposal based on the approach of learning by doing to develop training programs for public managers. First, we have analyzed the limitations of human resources processes, proposing competency models as an adequate framework for this purpose. Subsequently, after detecting training needs we have proposed a program that includes training and evaluative strategies for the so-called hard and soft competencies. Finally, we have discussed the implications of the training program in the current context of constant technological and social changes.
\end{abstract}

Keywords: competencies, public managers, training, organizational justice, blended learning.

\section{Introduction}

The recent economic crisis has revealed the breakdown suffered by the Spanish Public Administration (PA), that could be summarized in (Jiménez, 2018): (i) a public employment with a high average age being a major problem to face a digital transformation; (ii) a below desired level of technology in the information and communications era; and (iii) an anomie of values with a growing number of corruption cases, increasing the concern for the efficient provision of public services. Accordingly, the PA should aim for an improvement and modernization of its management practices (e.g., Instituto Nacional de Administración Pública - INAP, National Institute for Public Administration, 2014) in order to face the emerging organizational challenges for diversity, ethics, and digitalization, among others. For this purpose, given the relevant position of public managers due to the quality of their decisions, a crucial element is the vocational training for the development of their competencies (e.g., Berman \& West, 2003), and an effective way to achieve this is through vocational training. A learning modality useful for the vocational training of public managers is the blended learning. This learning modality allows to combine face-to- face and distance training and coaching through Massive Open Online Courses (MOOC) for the assurance of the development of competencies while reducing costs, making schedules more flexible, and bringing public managers closer to the use of the information and communications technology (e.g., Holland \& Piper, 2016; Singer \& Stoicescu, 2011; Singh, 2003). In this sense, the objective of this work is to elaborate a proposal based on the approach of learning by doing (Dewey, 1926; Schank, 2002) to develop vocational training programs for managers in the PA.

Taking into account the aforementioned objective, first, the limitations of human resources processes have been analyzed, and competency models have been proposed as an adequate framework for improving public management. Subsequently, we have detected current and future training needs, and a competency vocational training program has been proposed. Finally, the implications of the program inside the current context of constant technological and social changes have been discussed.

\section{Human resources challenges for the Spanish PA}

Public managers are those who perform managerial functions, according to Article 13 of the Spanish Real Decreto 
Legislativo 5/2015, de 30 de octubre, por el que se aprueba el texto refundido de la Ley del Estatuto Básico del Empleado Público (Royal Legislative Decree 5/2015 of October 30 approving the consolidated Basic Statute of Public Employees - BSPE). It is referred to both, positions where managerial functions are performed and are in charge of team workers, and high ranking public managers appointed on the basis of personal and political confidence criteria. These different types of public managers follow different access processes. On the one hand, public managers who access as civil servants face first public examinations, mainly by means of knowledge testing and/or combination of these with merit ratings. Then, promotion processes are similar to the access processes, nevertheless, two years of experience in the position are required. Regarding the limitations of these processes, when testing knowledge there is a perceived lack of connection between the contents and the tasks (Rodríguez, 2013), and the empirical evidence about its adequacy with the equality criterion is scarce (Salgado \& Moscoso, 2008). Regarding the merit ratings, Fondevila (2012) argues that they could violate the equality, merit, ability and fairness principles because: (i) politicians could favour their desired candidates by requiring specific merits (Ruano, Crespo, \& Polo, 2014), (ii) merit ratings indirectly discriminate women (Alonso, Táuriz, \& Choragwicka, 2009), and specifically, (iii) invasive privacy questions could lead to gender discrimination since they can be seen as activators of stereotypes (e.g., García-Izquierdo, Ramos-Villagrasa, \& Castaño, 2015).

On the other hand, some high-standing public managers can access the position through free assignment, since, for example, in many autonomous communities the regulation on the selection of their public managers has not yet been developed in detail (e.g., Areses et al., 2017). Here, another limitation emerges regarding the priority given to political loyalty over competencies (e.g., Bach \& Veit, 2017). As Џiménez (2009) notes, if the only selection criterion is the political confidence this greater discretion in the assignment may also imply a strained reading of the equality, fairness, merit and capacity principles. Moreover, the potential lack of professionalism in higher positions due to overlooking managerial competencies for the appointment of public managers could impact on management efficacy and decisions (e.g., Sánchez, 2018).

As a result of these limitations, the PA could be perceived as an organization with difficulties coping with the organizational challenges listed above. Thus, the Spanish PA must face its modernization by applying, among others, the lines of Open Government, transparency and equality in employment as an opportunity to restore the confidence of citizens.

\section{Open Government and equality}

Open Government is the application of principles concerned with accountability and the promotion of transparency, participation, and collaboration between citizenship and the PA in a fair way (García, 2014). In order to achieve an Open Government, several changes are required regarding some aspects as the following ones: (i) cultural, reminding that the main objective of the PA is serving citizens; (ii) within the organization which is designed as a hierarchical system not related to efficiency; and (iii) within the relationships between citizens and the PA, leading to more participative communication and a more open and transparent, facilitating the handling of information, thus requiring the development and use of technologies in order to communicate this information to the citizens.
On the other side, when workers perceive organizations as socially responsible, they respond with enhanced performance, citizenship, engagement, organizational attraction, and positive job attitudes (e.g., Rupp, Wright, Aryee, \& Luo, 2015). Consequently, the development of the Spanish Ley Orgánica 3/2007, de 22 de marzo, para la igualdad efectiva entre mujeres y hombres (Organic Law 3/2007 of March 22 about equal employment opportunities between women and men) and the BSPE aim to make effective the right to equal treatment and opportunities by eliminating discrimination against women in both private and public organizations. In the same vein, both Open Government and equal opportunities policies are closely related to preventing corruption and promoting ethics. This is because corruption in the PA not only occurs when the position is used to obtain a personal benefit, but also when there is a fraudulent mismanagement of the public resources prolonged in time, even if it does not seek own profit (Jareño, 2011). In this regard, Naessens (2010) points out that public employees with the proper competency training feel more confident to resist unethical behaviours related to corruption.

Faced with these challenges, traditional human resources management approaches are no longer sufficient, thus new approaches focused on competencies oriented towards a more volatile and changing context are required (e.g., MartínezGonzález, Selva, \& Crespo, 2019). In these new approaches it is worth highlighting transformational leadership (Bass, 1985), according to which management must create a change towards the personal development of employees within the organization; and the ethical leadership which encourages employees to adopt a repertoire of behaviours that prevent corruption (e.g., Stouten, van Dijke, \& De Cremer, 2012). More on this, according to Ramió and Salvador (2018), if the PA aims to be efficient and effective in the provision of services, it must have competent professionals with a vocation for public service. Consequently, it is necessary to identify new competency profiles and their assessment (e.g.. ఏiménez, 2018) by means of rigorous selection and promotion models that use reliable and valid evidence which can guarantee effective and fair evaluations in accordance with the principles of merit, capacity, publicity and equality (e.g., García-Izquierdo \& García-Izquierdo, 2007; García-Izquierdo, Moscoso, \& RamosVillagrasa, 2012; Rodríguez \& López-Basterra, 2018). Thus, a competency-based human resources model would facilitate the modernization of public institutions while offering an adequate framework for a successful and unbiased performance.

\section{Competency models}

A wide variety of definitions for the term competency have emerged since McClelland (1973) highlighted that competencies refer to a critical difference of behaviour of persons who have a successful performance. Going deeper into this, they are measurable characteristics of the behavioural repertoire that contribute significantly to job performance (e.g., Bartram, 2005; Salgado \& Cabal, 2011). According to Armstrong (2006), a competency-based model is useful for improving recruitment and selection, and performance management. Taking into account all these advantages, it is not surprising that several attempts to develop competency models both in public and private organizations have emerged (e.g., Ennis, 2008; United Kingdom Civil Service framework, 2015). This is also due, among other reasons, to the fact that competency models are an important tool in helping to define superior performance providing a common language for organizations (Briscoe \& Hall, 
1999). Furthermore, competency models may prove to be helpful for: (i) enhancing organizational justice (e.g., Cropanzano, Bowen, \& Gilliland, 2007); (ii) achieving face validity in evaluation processes (e.g., Truxillo \& Bauer, 2011); and (iii) reaching diversity (e.g., Castaño, Fontanil, \& García-Izquierdo, 2019) in human resources processes. Moreover, these models allow identifying the competencies required by public managers to guide their training (e.g., De Waal, 2010) when the position is accessed from lower ranks, where the responsibility is lower and the competences are very different (e.g., Park \& Faerman, 2018). This aspect is especially relevant in the case of the Spanish PA, given that according to Articles 14 and 69 of the BSPE, training is a right of public employees and a tool for contributing to provide services in an effective way, therefore, the proposal for a competency vocational training program for public managers will be presented next.

Table 1

Stages for developing a managerial competency vocational training program
Developing a competency vocational training program for public managers

Developing a competency vocational training program is a systematic process (Berrocal \& Pereda, 1999). In the case of public managers, firstly, training needs must be identified and detected in terms of competencies; secondly, training programs must be designed and carried out in accordance with training needs, establishing the learning modalities and methodologies best suited for public managers and their organizational context; and, thirdly, the program must be assessed. Table 1 provides a summary of these stages, which will be detailed below.

\begin{tabular}{|c|c|}
\hline Stage of the program & Description of the stages \\
\hline \multirow{3}{*}{$\begin{array}{l}\text { Identification and detection } \\
\text { of training needs }\end{array}$} & Steps for the identification and detection of training needs \\
\hline & $\begin{array}{l}\text { - Development of a preliminary competency model. } \\
\text { - Focus group sessions and Delphi panels with experts for refining the model. } \\
\text { - Job performance validity analysis. }\end{array}$ \\
\hline & Results: an eight-competency model \\
\hline \multirow{4}{*}{ Design and execution } & Modalities and methodologies \\
\hline & $\begin{array}{l}\text { - Initial assessment/certification by means of assessment centers. } \\
\text { - Training on competencies by means of blended-learning using MOOCs from active methodologies: } \\
\text { - Theoretical training: virtual classrooms. } \\
\text { - Distance training: simulations and problem solving; providing a channel for contacting mentors and } \\
\text { other participants (e.g., online meetings, messaging, forums). } \\
\text { - Face-to-face training: practical workshops and coaching by public managers with an extended career } \\
\text { know-how. }\end{array}$ \\
\hline & Other issues that should be considered \\
\hline & $\begin{array}{l}\text { - Training adapted to sight and hearing disability. } \\
\text { - Compliance with data privacy and labour risk prevention legislation. }\end{array}$ \\
\hline \multirow{4}{*}{ Assessment } & What information should be gathered and when? \\
\hline & $\begin{array}{l}\text { - Reactions, learning, behaviours, and results: immediately after the training and a while after the training } \\
\text { to analyse stability. } \\
\text { - Update of training needs and level of competencies: periodically after the training. }\end{array}$ \\
\hline & How to gather the information for the assessment? \\
\hline & $\begin{array}{l}\text { - Assessment centers, for competency levels, learning and behaviour. } \\
\text { - Satisfaction questionnaires, for the reactions of the participants. } \\
\text { - } 360^{\circ} \text { performance evaluations, number of complaints, satisfaction of subordinates, for the results. }\end{array}$ \\
\hline
\end{tabular}

\section{Identification and detection of vocational training needs}

The first stage for developing a vocational training program is the identification and detection of the training needs, which enables the implementation of the most efficient training strategies (Sladogna, 2003). In this regard, for the identification and detection of the competencies that managers need to develop, we propose to follow the hybrid approach of Briscoe and Hall (1999), which allows taking into account the current and future requirements of the organization combining qualitative and quantitative research. Table 2 shows some examples of the specific steps to develop models of managerial competencies that are framed in this hybrid approach. These examples have in common the participation of experts (i.e., human resources experts and employees with a deep knowledge of the job and the organization), the review of other competency models and the validation of the model in terms of work performance. 
Table 2

Summary of the main steps for the development of a managerial competency model

Draganidis \& Mentzas (2006)

(i) Creation of a team of experts.

(ii) Identification of performance metrics and validation sample.

(iii) Development of an initial competencies list through reviewing existing competency models.

(iv) Definition of competencies and behavioural indicators by conducting interviews, focus groups, and surveys; and (v) development of an initial competency model through quantitative and content analysis of the previous step.

(vi) Cross-check validation conducting additional focus groups, interviews, or surveys; and (vii) model refinement, performing quantitative and content analysis of the cross-check step.

(viii) Validation of the model, converting the competencies into a questionnaire.

(ix) Finalize the model, eliminating any competencies that do not correlate with the performance measures.
Czabanowska et al. (2013) and Tett, Guterman, Bleier, \& Murphy (2000)

(i) Literature and/or competency model review to obtain a preliminary competency model.

(ii) Refinement of the preliminary model with experts.
To better illustrate this phase of identification and detection, the following summarizes how a management competency model has been developed following the hybrid approach mentioned above within the research framework of the Evaluation and Selection of Public Managers project (ESPUMA project $\left.{ }^{1}\right)$. Firstly, a content analysis of the literature referring to public managerial competencies was carried out to obtain a preliminary competency model. Secondly, several focus group sessions with experts, and the completion of a questionnaire were accomplished for reviewing the preliminary model. Thirdly, the competency model was refined in a competency questionnaire by defining the competencies in terms of behavioural descriptors. Fourthly, the questionnaire was refined by carrying out a Delphi panel in which participants were asked to: (i) prioritize the competencies relating to public managerial performance reasoning their choices; (ii) indicate their degree of agreement with the competency list; (iii) indicate their agreement with the new list; and (iv) provide final suggestions. And fifthly, the psychometric properties of the competency questionnaire were analyzed and its job performance validity was successfully proved using a broad sample of public managers. As a result, an eight-competency model was developed, including: hard competencies such as planning, leadership, innovation orientation and civil servant orientation, and soft competencies such as recognition and regulation of emotions, engagement with the PA, communication, and ethics.

Once the competencies to be developed by the managers have been identified and detected, the next stage is the design and proposal of the training program for its development.

\section{Vocational training programs for public managers: a proposal}

Several vocational training programs for public managers have already been developed in Spain. The INAP has a specific program for public managers with managerial experience and those who are in pre-high managerial positions. Also the schools of business (e.g., IESE, ESADE), the Federación Española de Municipios y Provincias
(Spanish Federation of Municipalities and Provinces), and the PA schools of the autonomous communities offer training programs for public managers, besides the numerous Spanish universities which offer training programs for public management. However, despite the advantages of these programs, they also have some limitations. A first limitation refers to the voluntary nature of these programs. A second limitation refers to the loss of know-how of experienced managers due to the lack of knowledge management policies. A third limitation refers to the lack of a competency assessment prior to the start of the training to develop a customized program. For example, by means of the certification of competencies acquired during the exercise of managerial positions.

Over and above these limitations, a training program for the development of managerial competencies has been proposed below. This program is based on the model of the teachinglearning process suggested by de Miguel (2006), where the focus for planning the modalities, methods and assessment of the program is occupied by the competencies that must be developed.

First of all, the proposed vocational training program includes an initial stage for the assessment/certification of the competency background by means of behavioural assessments such as assessment centers, for instance. This assessment/certification includes situational judgment tests and work sample tests, among others, in which work environments are reconstructed to assess behaviours (e.g., Weekley, Hawkes, Guenole, \& Ployhart, 2015) and which are especially appropriate given their face and criterion validity (e.g., Anderson \& Witvliet, 2008; Christian, Edwards, \& Bradley, 2010). Carrying out an initial assessment/ certification of the competencies also enhances the suitability of the training program as it would be specifically focused on: (i) the competencies that are actually required from the training, (ii) the specific needs regarding every managerial level, and (iii) the specific needs regarding the PA level (i.e., local, autonomous, and state PA).

After this initial evaluation/certification, the proposal includes a training program from the blended-learning

For more information: https://espuma.uniovi.es/ 
modality, where the face-to-face modality is combined with autonomous work and distance-based training. This modality has demonstrated its effectiveness with respect to performance and satisfaction of participants (e.g., Ho, Lu, \& Thurmaier, 2006; Umek, Aristovnik, Tomaževič \& Keržič, 2015), as well as its suitability for strengthening teaching competencies (Singer \& Stoicescu, 2011). Moreover, blended learning contributes to opening the door to e-learning in the PA (Stoffregen, Pawlowski, \& Pirkkalainen, 2015) by taking advantage of both face-to-face learning and MOOC platforms. The flexibility of combining these two learning modalities also allows to include those who cannot attend courses at a specific time, and the learning from the on-the-job training approach, closer to the learning by doing approach. Regarding the methodologies for training (e.g., de Miguel, 2006), the proposed program follows a modular design which facilitates the execution of only those modules necessary based on the results of the initial evaluation/certification of competencies. However, this proposal is complemented by other methods especially suitable for the development of competencies where both fit, the lecture method, as well as other active methodologies such as case studies, tutorials, simulations, problem solving and serious games. These methodologies, which are in line with the idea of learning by doing, allows the training on how to face new situations successfully (e.g., Stene, 1970) by using new technologies for competency training in an attractive and challenging manner (Korteling, Helsdingen, \& Theunissen, 2013).

Last but not least, the vocational training program takes into account some other issues: (i) the materials and the MOOC are adapted to all the participants ensuring the equality principle in terms of accessibility, for example for those with sight disability and/or hearing impairment; (ii) moreover, the program must comply with the legislation on data privacy, especially when designing the MOOC platforms as well as when conducting the assessments; and (iii) closely related to this, the facilities for the training as well as the equipment for the distance training should provide the quality for an adequate training ensuring the compliance with the labour risk prevention legislation, also.

Taking into account the above, and following the example illustrated in the identification and detection of training needs section, the proposed training program for the identified competency model includes a blended-learning module for each competency, with a duration according to the case depending on the initial evaluation/certification and the level of competency development desired. The guiding principle of every module is to train participants to become a manager who for: (i) leadership: guides, influences and develops people, manages teams and collaborates and cooperates to achieve common objectives in line with transformational leadership approaches; (ii) ethics: behaves according to the principles of public service guaranteeing the rights of the people; (iii) recognition and regulation of emotions: shows emotional self-regulation, and the ability to detect their own and others emotions and takes them into account; (iv) engagement with the PA: understands the structure, objectives, functioning and policy of the organization, acting with loyalty; (v) communication: gives and obtains information in a clear, concise, timely and safe way; (vi) planning: establishes and manages realistic time-scales and resources to develop projects, achieves goals and manages an agenda; (vii) civil service orientation: helps and serves citizens by identifying and meeting the needs of citizenship; and finally, (viii) orientation innovation: develops and proposes actions, solutions and new forms of organization and improvements. On this basis, the training programme starts with a briefly theoretical training on the competency related behaviours by means of the MOOC platforms. For this purpose, the recording of live events and virtual classrooms are helpful. Then, active learning methodologies from the learning by doing approaches, such as simulations and problem solving are used for distance training. All of these always providing a channel when doubts arise for contacting mentors by means of online meetings, messaging and discussion forums with other participants. As for the simulations, situational judgment tests and work samples are used, which are complemented by the use of serious games and problem solving activities. Finally, distance training is complemented with face-to-face sessions by means of practical workshops where participants could meet other participants to share and discuss working experiences; and also by means of coaching by public managers with an extended career know-how.

\section{Assessing the vocational training program}

Finally, after the training program, the next stage is its assessment, for which it is proposed to take into account what information should be collected, when and how to collect such information.

Regarding the what and when, on the one hand, following the levels of the New World Kirkpatrick Model (Kirkpatrick \& Kirkpatrick, 2016), the assessment of the following aspects is proposed: (i) reactions, that is, the degree to which participants find the training favourable, engaging and relevant; (ii) learning, that is, the degree to which participants acquire the intended knowledge, skills, attitude, confidence and commitment; (iii) behaviour, that is, the degree to which participants apply on the job what they learned and (iv) results, that is, the degree to which outcomes occur as a result of the training. In order to obtain information on these four aspects, it is recommended to make an assessment after carrying out the training program and repeat it after a while to analyze the stability of the changes obtained with the program. In addition, it would be advisable to assess the learning, behaviour and results also before carrying out the program in order to assess the extent to which the training has been effective.

On the other hand, the suitability of the program should be assessed periodically to evaluate the possibility of modifying its modality, learning methodologies and the competencies to be developed given the current context of continuous changes. Likewise, it is not enough for public managers to participate in the training program once throughout their professional career, but it is proposed to carry out periodic assessments of their level of competencies to keep the training updated, guaranteeing their professional development over time. Ultimately, the assessment of the training program must be useful not only to value its effectiveness, but also to guide future training programs by improving the willingness of public managers to participate (e.g., Dysvik \& Kuvaas, 2008) while ensuring the correction of problems and the anticipation of the new training needs.

Dealing with how to gather the above-mentioned information, assessment centers, situational judgment tests, and work sample tests are appropriate for the learning and behavioural assessment. Also, questionnaires dealing with the training contents, the length, and the learning methodologies are adequate for measuring the reactions towards the program. Moreover, $360^{\circ}$ performance assessments, the analysis of the number of citizens' complaints, and the satisfaction of subordinates in terms of the managerial work, among others, are useful for assessing results.

Returning to the example of the proposed training program of the previous section, its assessment comprises two phases. 
A phase for assessing the reactions, the learning, the behaviour and the results, and a phase of periodic review of the training program and the levels of competencies of public managers. For the first phase, assessment centers, situational judgment tests and work samples specifically designed for the assessment of competencies are used. This assessment is carried out before and after carrying out the training program and is repeated after some time for comparison. In the same way, $360^{\circ}$ performance assessments, as well as the analysis of the satisfaction of the subordinates with public managers, and the number of complaints by the citizens regarding the provision of public services are used before and after carrying out the program, and also after a while. Finally, immediately after finishing the program, satisfaction questionnaires with training are applied. The second phase includes a periodic review of the modalities and methodologies used. For this, the training program is reviewed in line with the new learning methodologies and modalities, the technological developments, and the new training needs in terms of managerial competences. This review is complemented by a periodic evaluation of the levels of competencies of public managers who have already received training for the planning of the reinforcement training and the updating of competencies.

\section{Discussion and conclusions}

In this work, a training program for public managers has been proposed based on the competencies required for an effective management in the face of the organizational challenges of the PA, and therefore which can contribute to its modernization. Thus, the main characteristics of the training program proposed here will be discussed below.

Firstly, it is important to highlight the relevance of the identification and detection of needs stage and its updating over time, which must take into account not only the current situation, but also the future technological and social changes for defining the contents of the vocational training program in terms of managerial competencies (i.e., ethics, leadership, innovation orientation).

Secondly, the initial assessment/certification of competencies reduces times and costs, adjusting the program tailored to specific needs.

Thirdly, in line with the above, given the particular characteristics of public managers, the blended learning together with the active training methodologies allow the program to be adapted as best as possible to the needs of the public managers for the effectiveness of the on-the-job training. In addition, the use of MOOC allows public managers to approach new technologies, which in turn brings them closer to the principles of the Open Government. Besides, the flexibility of blending gives women the possibility to access to the vocational training considering motherhood periods and family responsibilities (e.g., Castaño et al., 2019). Then, the competency vocational training model would allow women and men equal training for accessing management positions contributing to the fulfilment of the equality and justice principles.

In conclusion, the implementation of the training program based on blended learning proposed here can result in the modernization of the PA (e.g., Moskal, Dziuban, \& Hartman, 2013) through the flexibility to adapt to organizational and social changes, the saving of time and costs due to its tailor-made adaptation, the application of new technologies in teachinglearning models, and the use of active methodologies for the onthe-job training of the required competencies.
Funding: The authors disclosed receipt of the following financial support for the research, authorship and/or publication of this article: Ministerio de Economía y Competitividad and Fondos Sociales Europeos (project reference PSI-2013-44854R), Cátedra Asturias Prevención (CATI-04-2018), and Consejería de Economía y Empleo del Gobierno del Principado de Asturias and Fondos Europeos de Desarrollo Regional (reference FC-GRUPIN-IDI/2018/000132).

\section{Referencias / References}

Aguinis, H. (2011). Organizational responsibility: Doing good and doing well. En S. Zedeck (Ed.), APA handbook of industrial and organizational psychology (pp. 855-879). Washington, DC: American Psychological Association.

Alonso, P., Táuriz, G., \& Choragwicka, B. (2009). Valoraciones de Méritos (Training and Experience) en la Administración Pública y la Empresa: Fiabilidad, Validez y Discriminación de Género. Journal of Work and Organizational Psychology, 25(3), 245-258. Recuperado de http://scielo.isciii.es/scielo.php?script=sci_arttext\&pi$\mathrm{d}=$ S1576-59622009000300005

Anderson, N., \& Witvliet, C. (2008). Fairness Reactions to Personnel Selection Methods: An international comparison between the Netherlands, the United States, France, Spain, Portugal, and Singapore. International Journal of Selection and Assessment, 16(1), 1-13. doi: https://doi.org/10.1111/j.1468-2389.2008.00404.x

Areses, X., Blanco, C., Colmenares, P., Fernández, E., Gallego, A., Gómez, G., Íniguez, S., Labrada, M. L., Mapelli, C., Pastor, I., \& Souto, J. (2017). Nuevos tiempos para la función pública: Propuestas para atraer y desarrollar el talento en la Administración General del Estado. Madrid: INAP.

Armstrong, M. (2006). A Handbook of Human Resource Management Practice. London: Kogan Page.

Bach, T., \& Veit, S. (2017). The Determinants of Promotion to High Public Office in Germany: Partisan Loyalty, Political Craft, or Managerial Competencies? Journal of Public Administration Research and Theory, 28(2), 254-269. doi: https://doi.org/10.1093/jopart/mux041

Bartram, D. (2005). The Great Eight Competencies: A Criterion-Centric Approach to Validation. Journal of Applied Psychology, 90(6), 11851203. doi: http://doi.org/10.1037/0021-9010.90.6.1185

Bass, B. M. (1985). Leadership and performance beyond expectations. Nueva York: Free Press.

Berman, E. M., \& West, J. P. (2003). Solutions to the problem of managerial mediocrity: Moving Up to Excellence (Part 2). Public Performance \& Management Review, 27(2), 30-52. doi: https://doi.org/10. 1080/15309576.2003.11051791

Berrocal, F., \& Pereda, S. (1999). La psicología del trabajo y la formación en la empresa. Revista complutense de educación, 10(1), 37-59. Recuperado de https://dialnet.unirioja.es/servlet/articulo?codigo $=150265$

Briscoe, J. P., \& Hall, D. T. (1999). An alternative approach and new guidelines for practice. Organizational Dynamics, 28(2), 37-52. doi: http://doi.org/10.1016/S0090-2616(00)80015-7

Castaño, A. M., Fontanil, Y., \& García-Izquierdo, A. L. (2019). "Why Can't I Become a Manager?”-A Systematic Review of Gender Stereotypes and Organizational Discrimination. International Journal of Environmental Research and Public Health, 16(10), 1813. doi: https://doi.org/10.3390/ijerph16101813

Christian, M. S., Edwards, B. D., \& Bradley, J. C. (2010). Situational judgment tests: Constructs assessed and a meta-analysis of their criterion related validities. Personnel Psychology, 63(1), 83-117. doi: https://doi.org/10.1111/j.1744-6570.2009.01163.x

Cropanzano, R., Bowen, D. E., \& Gilliland, S. W. (2007). The management of organizational justice. Academy of Management Perspectives, 21(4), 34-48. doi: http://dx.doi.org/10.5465/AMP.2007.27895338 
Czabanowska, K., Smith, T., Könings, K. D., Sumskas, L., Otok, R., Bjegovic-Mikanovic, V., \& Brand, H. (2013). In search for a public health leadership competency framework to support leadership curriculum-a consensus study. The European Journal of Public Health, 24(5), 850-856. doi: http://doi.org/10.1093/eurpub/ckt158

de Miguel, M. (2006). Modalidades de enseñanza centradas en el desarrollo de competencias. Oviedo: Ediciones Universidad de Oviedo. Recuperado de http://www.uniovi.net/zope/servicios/comunidad/ publicaciones/catalogo/titulos/i539/

de Waal, A. A. (2010). Achieving High Performance in the Public Sector. Public Performance \& Management Review, 34(1), 81-103. doi: https://doi.org/10.2753/PMR1530-9576340105

Dewey, J. (1926). Democracy and education. New York: MacMillan.

Draganidis, F., \& Mentzas, G. (2006). Competency based management: a review of systems and approaches. Information $\mathrm{Ma}$ nagement \& Computer Security, 14(1), 51-64. doi: http://doi. org/10.1108/09685220610648373

Dysvik, A., \& Kuvaas, B. (2008). The relationship between perceived training opportunities, work motivation and employee outcomes. International Journal of Training and Development, 12(3), 138-157. doi: https://doi.org/10.1111/j.1468-2419.2008.00301.x

Ennis, M. R. (2008). Competency Models: A Review of the Literature and The Role of the Employment and Training Administration (ETA). U. S. Department of labor. Recuperado de http://www.careeronestop. org/CompetencyModel/competency-models/pyramid-home.aspx

Fondevila, J. (2012). Manual para la selección de empleados públicos. Madrid: El consultor de los Ayuntamientos (La Ley).

García, J. (2014). Gobierno abierto: transparencia, participación y colaboración en las administraciones públicas. Innovar, 24(54), 75-88. doi: http://dx.doi.org/10.15446/innovar.v24n54.46441

García-Izquierdo, A. L., \& García-Izquierdo, M. (2007). Discriminación, igualdad de oportunidades en el empleo y selección de personal en España. Journal of Work and Organizational Psychology, 23, 111-138. Recuperado de http://www.redalyc.org/articulo. oa?id=231317574007

García-Izquierdo, A. L., Moscoso, S., \& Ramos-Villagrasa, P. J. (2012). Reactions to the Fairness of Promotion Methods: Procedural justice and job satisfaction. International Journal of Selection and Assessment, 20(4), 394-403. doi: http://dx.doi.org/10.1111/ijsa.12002

García-Izquierdo, A. L., Ramos-Villagrasa, P. J., \& Castaño, A. M. (2015). e-Recruitment, gender discrimination, and organizational results of listed companies on the Spanish Stock Exchange. Journal of Work and Organizational Psychology, 31(3), 155-164. doi: http:// dx.doi.org/10.1016/j.rpto.2015.06.003

Ho, A., Lu, L., \& Thurmaier, K. (2006). Testing the Reluctant Professor's Hypothesis: Evaluating a Blended-Learning Approach to Distance Education. Journal of Public Affairs Education, 12(1), 81-102. Recuperado de https://www.jstor.org/stable/40215727

Holland, D. D., \& Piper, R. T. (2016). High-Trust Leadership and Blended Learning in the Age of Disruptive Innovation: Strategic Thinking for Colleges and Schools of Education. Journal of Leadership Education, 15(2), 74-97. doi: http://doi.org/10.12806/V15/I2/T2

Instituto Nacional de Administración Pública - INAP. (2014). Panorama de las Administraciones Públicas 2013. Madrid: INAP.

Jareño, A. (2011). Corrupción y delincuencia de los funcionarios en la contratación pública. Madrid: Iustel Publicaciones.

Jiménez, R. (2009). El personal directivo en la administración local. Recuperado de http://cemical.diba.cat/es/publicaciones/ficheros/CEMICAL_personal_directivo_cast.pdf

Jiménez, R. (2018). Función pública (II): Efectos de la crisis. Recuperado de https://rafaeljimenezasensio.com/2018/02/18/funcion-publicaii-efectos-de-la-crisis/

Korteling, H., Helsdingen, A., \& Theunissen, N. C. M. (2013). Serious gaming@ work: Learning job-related competencies using serious gaming. En A. Bakker \& D. Derks (Eds.), The Psychology of Digital Media at Work (pp. 123-144). London: Psychology Press. doi: https://doi.org/10.4324/9780203074145-12

Ley Orgánica 3/2007, de 22 de marzo, para la igualdad efectiva entre mujeres y hombres. Boletín Oficial del Estado de 23 de marzo de 2007. Recuperado de https://www.boe.es/diario_boe/txt.php?id=BOE-A-2007-6115

Martínez-González, M., Selva, C., \& Crespo, J. L. (2019). La evolución de las competencias de RRHH en organizaciones inmersas en la cuarta revolución industrial. Quaderns de Psicologia, 21(1), 1471. doi: https://doi.org/10.5565/rev/qpsicologia.1471

McClelland, D. C. (1973). Testing for competence rather than for "intelligence”. American Psychologist, 28(1), 1-14. doi: http://dx.doi. org/10.1037/h0034092

Moskal, P., Dziuban, C., \& Hartman, J. (2013). Blended learning: A dangerous idea? The Internet and Higher Education, 18, 15-23. doi: https://doi.org/10.1016/j.iheduc.2012.12.001

Kirkpatrick, J. D., \& Kirkpatrick, W. K. (2016). Kirkpatrick's four levels of training evaluation. Alexandria: ATD Press.

Naessens, H. (2010). La ética pública y su proyección en el valor de la transparencia. En L. Peña, T. Ausín, \& O. Diego (Eds.), Ética y servicio público (pp. 332-362). Madrid: Plaza y Valdés.

Park, H. H., \& Faerman, S. (2019). Becoming a Manager: Learning the Importance of Emotional and Social Competence in Managerial Transitions. The American Review of Public Administration, 49(1), 98-115. doi: https://doi.org/10.1177/0275074018785448

Ramió, C., \& Salvador, M. (2018). La Nueva Gestión del Empleo Público. Recursos Humanos e Innovación de la Administración. Barcelona: Tibidabo Ediciones, S. A.

Real Decreto Legislativo 5/2015, de 30 de octubre, por el que se aprueba el texto refundido de la Ley del Estatuto Básico del Empleado Público. Boletín Oficial del Estado de 31 de octubre de 2015. Recuperado de https://www.boe.es/buscar/act.php?id=BOE-A-2015-11719

Rodríguez, A. (2013). Planificación de Recursos Humanos en las Administraciones Públicas. Gestión y desarrollo de personas en tiempos de austeridad. Madrid: INAP.

Rodríguez, A., \& López-Basterra, J. (2018). Predictores Selectivos en el Sector Público: Validez Predictiva y Reacciones de los Candidatos. Journal of Work and Organizational Psychology, 34(1), 16-28. doi: http://dx.doi.org/10.5093/jwop2018a3

Ruano, J. M., Crespo, J., \& Polo, C. (2014). Los funcionarios ante el espejo. Análisis del ciclo de la selección de personal en la Administración General del Estado. Madrid: INAP.

Rupp, D. E., Wright, P. M., Aryee, S., \& Luo, Y. (2015). Organizational Justice, Behavioral Ethics, and Corporate Social Responsibility: Finally the Three Shall Merge. Management and Organization Review, 11(1), 15-24. doi: http://dx.doi.org/10.1017/mor.2015.8

Salanova, M., \& Grau, R. M. (1999). Análisis de necesidades formativas y evaluación en contextos de cambio tecnológico. Revista de psicología general y aplicada: Revista de la Federación Española de Asociaciones de Psicología, 52(2-3), 329-350. Recuperado de https:// dialnet.unirioja.es/servlet/articulo? codigo $=2498356$

Salgado, J. F., \& Cabal, A. L. (2011). Evaluación del desempeño en la Administración Pública del Principado de Asturias: Análisis de las propiedades psicométricas. Journal of Work and Organizational Psychology, 27(2), 75-91. doi: http://dx.doi.org/10.5093/tr2011v27n2a1

Salgado, J. F., \& Moscoso, S. (2008). Selección de personal en la empresa y las AAPP: de la visión tradicional a la visión estratégica. Papeles del Psicólogo, 29(1), 16-24. Recuperado de http://www.redalyc.org/ $\mathrm{html} / 778 / 77829103 /$

Sánchez, M. (2018). Las Administraciones Españolas. Madrid: Tecnos.

Schank, R. C. (2002). Designing World-Class e-Learning: How IBM, GE, Harvard Business School, and Columbia University are Succeeding at e-Learning. New York: McGraw-Hill. 
Singer, F. M., \& Stoicescu, D. (2011). Using blended learning as a tool to strengthen teaching competences. Procedia Computer Science, 3, 1527-1531. doi: https://doi.org/10.1016/j.procs.2011.01.043

Singh, H. (2003). Building Effective Blended Learning Programs. Educational Technology, 43(6), 51-54. Recuperado de https://eric.ed.go$\mathrm{v} /$ ?id=EJ679022

Sladogna, M. G. (2003). ¿La empresa como espacio formativo? Repensar la formación para y en el trabajo. Boletín Cinterfor: Boletín Técnico Interamericano de Formación Profesional, 154, 9-34. Recuperado de https://dialnet.unirioja.es/servlet/articulo? codigo $=1007238$

Stene, E. O. (1970). Relevance of Training for Public Administration. Midwest Review of Public Administration, 4(2), 87-89. doi: https:// doi.org/10.1177/027507407000400205

Stoffregen, J., Pawlowski, J. M., \& Pirkkalainen, H. (2015). A Barrier Framework for open E-Learning in public administrations. Computers in Human Behavior, 51, 674-684. doi: https://doi.org/10.1016/j. chb.2014.12.024

Stouten, J., van Dijke, M., \& De Cremer, D. (2012). Ethical Leadership: An Overview and Future Perspectives. Journal of Personnel Psychology, 11(1), 1-6. doi: http://dx.doi.org/10.1027/1866-5888/a000059
Tett, R. P., Guterman, H. A., Bleier, A., \& Murphy, P. J. (2000). Development and Content Validation of a «Hyperdimensional» Taxonomy of Managerial Competence. Human Performance, 13(3), 205-251. doi: http://doi.org/10.1207/S15327043HUP1303_1

Truxillo, D. M., \& Bauer, T. N. (2011). Applicant reactions to organizations and selection systems. En S. Zedeck (Ed.), APA Handbook of I/O Psychology (Vol. 2, pp. 379-397). Washington, DC: American Psychological Association Press.

Umek, L., Aristovnik, A., Tomaževič, N., \& Keržič, D. (2015). Analysis of Selected Aspects of Students' Performance and Satisfaction in a Moodle-Based E-Learning System Environment. Eurasia Journal of Mathematics, Science and Technology Education, 11(6), 1495-1505. doi: https://doi.org/10.12973/eurasia.2015.1408a

United Kingdom Civil Service. (2015). Civil Service: Competency Framework 2012-2017. Recuperado de https://www.gov.uk/government/uploads/system/uploads/attachment_data/file/436073/cscf_ fulla4potrait_2013-2017_v2d.pdf

Weekley, J. A., Hawkes, B., Guenole, N., \& Ployhart, R. E. (2015). Low-Fidelity Simulations. Annual Review of Organizational Psychology and Organizational Behavior, 2(1), 295-322. doi: https://doi. org/10.1146/annurev-orgpsych-032414-111304 